\title{
The psychological aftermath of prostate cancer treatment choices: a comparison of depression, anxiety and quality of life outcomes over the 12 months following diagnosis
}

\author{
Jeremy W Couper, Anthony W Love, Judith V Dunai, Gillian M Duchesne, Sidney Bloch, Anthony J Costello and David W Kissane
}

$\mathrm{P}$ rostate cancer (PCA) represents a major health issue in Australia but, unlike breast cancer in women, little is known about the psychological impact of this cancer and its treatments in men. ${ }^{1}$ PCA is the most commonly occurring cancer and second leading cause of cancer death in Australian men; however, most men survive for a decade or more following initial diagnosis. ${ }^{2}$ For such men and their doctors, there is currently a lack of clear guidance from the scientific literature about which of the available PCA treatments offers the best survival/side-effect balance or which clinical factors in the patient should favour the choice of one treatment over another. ${ }^{3}$

The main treatments for localised PCA are: - Active surveillance/watchful waiting (WW), which involves no active treatment but may entail repeated rectal examinations, prostate-specific antigen estimations and repeat prostate biopsies;

- Radical prostatectomy (RP), which involves complete surgical removal of the prostate gland, seminal vesicles, ampulla of vas deferens and possibly pelvic lymph nodes; - Androgen ablation or "hormone therapy" (HT), which involves oral or injected drugs to lower circulating androgen levels or block their action (surgical removal of testes is now rarely employed); and

- Other early treatments (OET), which primarily include external beam radiotherapy (EBRT), where multiple doses of radiation are applied to the prostate gland from an external source over several weeks, or brachytherapy, in which low-dose permanent or high-dose temporary radioactive implants are inserted into the prostate. ${ }^{4}$

Because each of the active treatment choices outlined above (RP, HT and OET) entail troublesome side effects that can have an enduring impact on physical and psychological wellbeing, studies that compare groups of patients who receive different treatments are needed. There is growing recognition of the importance of healthrelated quality of life (HRQOL) and psychological wellbeing as pertinent considerations in addition to patient survival time when deciding on PCA treatment. ${ }^{5}$

\section{ABSTRACT}

Objective: To assess the psychological impact of the different treatments for localised prostate cancer (PCA).

Design, participants and setting: Observational, prospective study of consecutive patients with PCA attending clinics in public hospitals and private practices in metropolitan Melbourne between 1 April 2001 and 30 December 2005. Data were collected at initial diagnosis of histologically confirmed localised PCA, and close to the commencement of definitive treatment (Time 1), and 12 months later (Time 2). Patients were stratified according to treatment type (radical prostatectomy [RP], hormone therapy $[\mathrm{HT}]$ or other early treatment including radiation therapies [OET]). Patients who elected to undergo active surveillance/watchful waiting (WW) rather than active treatment were treated as a naturalistic control group.

Main outcome measures: Levels of depression and anxiety were assessed by the Brief Symptom Inventory, and physical and psychosocial aspects of health-related quality of life (HRQOL) were assessed by the 36-item Short-Form Health Survey.

Results: 211 patients with PCA were recruited; 193 completed the Time 1 questionnaires ( $38 \mathrm{RP}, 56 \mathrm{HT}, 38 \mathrm{OET}$ and $61 \mathrm{WW}$ ); and 172 completed the Time 2 questionnaires ( $33 \mathrm{RP}, 51 \mathrm{HT}, 33 \mathrm{OET}$ and $55 \mathrm{WW}$ ). At Time 1, the three active treatment groups all reported greater dysfunction in work role and daily activities compared with the WW group. The RP group also reported worse social and emotional role functioning, while the HT and OET groups reported poorer vitality levels. The HT group reported significantly higher depression scores. At Time 2, the RP and OET groups did not differ from the WW group on either HRQOL or psychological status. By contrast, the HT group reported significantly worse $\mathrm{HRQOL}$ (physical functioning, role-physical and vitality domains) and greater psychological distress compared with the WW group.

Conclusions: Compared with the other active treatments for localised PCA, HT appears to be associated with poorer $\mathrm{HROOL}$ and greater psychological distress 12 months after commencing treatment.

MJA 2009; 190: S86-S89

Here, we use data from our 2001-2005 observational study of patients with localised PCA, ${ }^{6}$ collected at the time of diagnosis and initial treatment decision and at 12 months later, to compare psychological status (depression and anxiety) and HRQOL as a function of treatment type at these temporal points.

\section{METHODS}

Our multisite study was designed to elicit data from two cohorts of patients facing PCA: (i) patients who had just learned they had localised (potentially curable) PCA; and (ii) patients who had been told they had metastatic (incurable) PCA. ${ }^{6}$ The data reported here are from the localised PCA cohort.
Patients were consecutive attendees at participating clinics in public hospitals and private practices in metropolitan Melbourne between 1 April 2001 and 30 December 2005 , and were recruited into the study by their oncologists or urologists. Eligible patients had to have histologically confirmed PCA confined to the prostate gland (ie, no evidence of lymph node involvement or metastasis). Exclusion criteria included inability to give informed consent, diagnosis of another cancer, minimal understanding of English, psychosis, intellectual disability or dementia.

Each patient gave written consent to participate. Institutional ethics approval was obtained from human research ethics committees at each of the seven hospitals 
involved in the study and from the University of Melbourne.

Patients completed self-report questionnaires before initiation of definitive PCA treatment, or as soon as practicable afterwards (Time 1), and again 12 months later (Time 2). Demographic data were collected from all patients. Where geographically possible, Time 1 questionnaires were administered in the patient's home. Care was taken to ensure that partners and other family members were not present. After initial telephone contact to reinforce the

\section{Sociodemographic characteristics of the sample of 211 participants at the time of recruitment}

\begin{tabular}{lc} 
Characteristic & Value \\
\hline Age (years) & \\
Mean (SD) & $66.15(8.26)$ \\
Range & $43-92$ \\
Marital status & \\
Married/de facto & $78.3 \%$ \\
Single/widowed/divorced/ & $21.7 \%$ \\
separated & \\
Occupational status & \\
Employed & $36.8 \%$ \\
Retired/unemployed/ & $63.2 \%$ \\
pensioner &
\end{tabular}

importance of completing the questionnaires independently of family members, Time 2 questionnaires were sent by mail.

\section{Measures}

- The 36-item Short-Form Health Survey $(\mathrm{SF}-36)^{7}$ is a well validated and reliable selfreport measure both of physical and psychosocial aspects of HRQOL. ${ }^{8}$ It has eight subscales - four covering physical health, and four relating to mental health. There are four physical health subscales - "physical function", "role-physical", "pain" and "general health". "Role-physical" refers to performance of work or other daily activities without interference from physical impediments. "Vitality" refers to the balance of energy and fatigue levels. "Social functioning" is related to performance of normal social activities without interference from physical or emotional problems. "Role-emotional" refers to performance of work or other daily activities without interference from emotional or psychological impediments. "Mental health" is related to the balance between feelings of nervousness and depression and feeling peaceful, happy and calm. Respondents are asked to report on their HRQOL over the 4 weeks before the day the questionnaire is administered. Lower scores on all subscales indicate poorer functioning.

- The Brief Symptom Inventory (BSI-53) ${ }^{9}$ is a 53-item self-report measure of psycho- logical status that has been extensively used in psycho-oncology research. In addition to an overall scale for general psychological distress, it provides nine subscales, including ones for depression and anxiety that are reported here. In each case, a higher score denotes greater psychological distress. Respondents are asked to report on their psychological status over the week before the day the questionnaire is administered. The BSI-53's ability to discriminate clinically distressed from non-distressed samples has been well established, using structured psychiatric interviews as benchmarks. It also has well established reliability and convergent and predictive validity. ${ }^{10}$ It is sensitive to changes in psychological adjustment and to psychopathology in populations of patients with cancer. $^{11,12}$

\section{Statistical analysis}

In the data presented below, we cite the number of participants at Time 1 and Time 2 who had complete data. SPSS, version 14.0 (SPSS Inc, Chicago, Ill, USA) was used throughout. Analyses comprised planned comparisons between WW and each of the other three treatments (RP, HT and OET), based on the assumption that without active treatment, the men in the WW condition would form a naturalistic control or comparison group over time. Planned compar-

\section{Depression, anxiety and health-related quality of life at diagnosis (Time 1) and at 12 months (Time 2) in patients with prostate cancer, assessed by scores (mean [SD]) on subscales of the Brief Symptom Inventory and the 36-item Short-Form Health Survey}

\begin{tabular}{|c|c|c|c|c|c|c|c|c|c|c|c|}
\hline \multirow[b]{2}{*}{ Treatment } & \multirow[b]{2}{*}{$\begin{array}{c}\text { No. of } \\
\text { patients }\end{array}$} & \multicolumn{2}{|c|}{$\begin{array}{l}\text { Brief Symptom } \\
\text { Inventory subscale }\end{array}$} & \multicolumn{8}{|c|}{ 36-item Short-Form Health Survey subscale } \\
\hline & & Depression & Anxiety & $\begin{array}{l}\text { Physical } \\
\text { function }\end{array}$ & $\begin{array}{l}\text { Role- } \\
\text { physical }\end{array}$ & Pain & $\begin{array}{l}\text { General } \\
\text { health }\end{array}$ & Vitality & $\begin{array}{c}\text { Social } \\
\text { function }\end{array}$ & $\begin{array}{l}\text { Role- } \\
\text { emotional }\end{array}$ & $\begin{array}{l}\text { Mental } \\
\text { health }\end{array}$ \\
\hline \multicolumn{12}{|l|}{$\overline{T i m e} 1$} \\
\hline WW & 61 & $0.147(0.3)$ & $0.246(0.4)$ & $85.66(21.5)$ & $90.98(25.0)$ & $89.84(17.0)$ & $72.29(19.0)$ & $75.08(15.5)$ & $89.14(16.0)$ & $87.43(28.6)$ & $80.59(16.5)$ \\
\hline $\mathrm{RP}$ & 38 & $0.289(0.4)$ & $0.307(0.6)$ & $79.47(22.9)$ & $55.26(45.1)^{\star}$ & $84.20(18.6)$ & $77.16(15.7)$ & $68.55(18.6)$ & $75.99(25.6)^{\dagger}$ & $69.30(42.0)^{\dagger}$ & $78.53(17.0)$ \\
\hline HT & 56 & $0.348(0.6)^{\ddagger}$ & $0.360(0.5)$ & $79.29(18.5)$ & $62.95(40.2)^{\star}$ & $83.94(18.2)^{\S}$ & $66.20(20.7)$ & $57.60(21.2)^{\star}$ & $81.70(23.6)$ & $79.17(35.1)$ & $74.71(19.5)^{\S}$ \\
\hline OET & 38 & $0.246(0.4)$ & $0.237(0.4)$ & 79.47 (21.4) & $68.42(37.1)^{\star}$ & 85.55 (17.9) & $64.82(19.9)$ & $66.45(19.9)^{\ddagger}$ & $83.88(22.5)$ & 77.19 (35.6) & 77.89 (15.4) \\
\hline All & 193 & $0.253(0.5)$ & $0.289(0.5)$ & $81.37(21.0)$ & 71.37 (38.9) & $86.17(17.9)$ & $70.01(19.5)^{\S}$ & $67.02(19.8)$ & $83.35(22.0)$ & 79.45 (35.2) & 77.95 (17.3) \\
\hline \multicolumn{12}{|l|}{ Time 2} \\
\hline WW & 55 & $0.169(0.3)$ & $0.151(0.2)$ & $83.91(19.8)$ & 74.09 (39.9) & 84.41 (19.8) & $71.41(20.6)$ & $67.09(19.5)$ & $85.68(21.8)$ & $80.61(34.9)$ & $82.54(14.6)$ \\
\hline $\mathrm{RP}$ & 33 & $0.252(0.4)$ & $0.258(0.4)$ & $86.67(17.7)$ & 71.97 (36.3) & $80.74(21.3)$ & $74.93(17.2)$ & 70.15 (17.5) & $87.50(20.0)$ & $87.88(23.3)$ & $78.67(14.5)$ \\
\hline $\mathrm{HT}$ & 51 & $0.355(0.5)^{\ddagger}$ & $0.291(0.4)^{\ddagger}$ & $66.76(21.8)^{\star}$ & $50.00(42.1)^{\dagger}$ & $76.64(21.6)^{\S}$ & $64.06(22.2)^{\S}$ & $56.18(18.7)^{\dagger}$ & $81.13(22.4)$ & $75.82(36.6)$ & 78.35 (15.4) \\
\hline OET & 33 & $0.219(0.4)$ & $0.200(0.3)$ & $80.76(19.3)$ & $75.76(36.2)$ & $82.40(19.6)$ & $66.76(21.8)$ & $62.27(19.7)$ & $86.74(21.6)$ & 84.85 (30.1) & $81.70(16.3)$ \\
\hline All & 172 & $0.250(0.4)$ & $0.222(0.3)$ & $78.75(21.3)$ & $66.86(40.4)$ & $81.02(20.7)$ & $69.01(20.9)$ & $63.51(19.5)$ & $84.88(21.6)$ & 81.39 (32.7) & $80.39(15.2)$ \\
\hline
\end{tabular}


isons were conducted by univariate analysis of variance (with an alpha level of 0.05).

\section{RESULTS}

Referring oncologists and urologists at the multiple clinical sites identified 211 eligible patients with localised PCA from clinic attendees. Their demographic data are shown in Box 1. Of these 211 patients, 193 completed the Time 1 questionnaires and 172 (89.1\% of those at Time 1) completed the Time 2 questionnaires.

\section{Time 1}

At Time 1, the three active treatment groups all experienced more dysfunction compared with the WW group (see Box 2); this was particularly evident in their scores on the role-physical subscale of the SF-36. In addition, the men in the RP group reported more disruption to their social functioning and emotional role. The HT and OET groups reported poorer vitality levels. The HT group had significantly worse depression scores than the WW group (see Box 2).

\section{Time 2}

At Time 2, the HRQOL and psychological status of the RP and OET groups had become very similar to those of the WW group. In contrast, the HRQOL and psychological status of the HT group appeared to have deteriorated, with significantly worse HRQOL with respect to physical functioning, physical role and vitality, and greater psychological distress in comparison to the WW group (see Box 2). This pattern was most evident for depression (see Box 2).

\section{DISCUSSION}

Our study is unusual in that we: (i) recruited patients newly diagnosed with PCA who were receiving all the main types of PCA treatment; (ii) prospectively measured their HRQOL and psychological status; and (iii) compared these outcomes between the treatment groups after a clinically meaningful period of time had elapsed.

We found that patients in the three active treatment groups (RP, HT and OET) experienced greater dysfunction initially compared with those in the WW group, but the HT group alone demonstrated continuing poor HRQOL with respect to physical functioning, role-physical and vitality 12 months later. In addition, the HT group alone experienced an increase in psychological distress in comparison to the WW group 12 months after their treatment for PCA started. To our knowledge, this is the first prospective study of localised PCA to demonstrate enduring adverse psychological effects of HT.

Few studies have examined psychosocial outcome as a function of differential treatment for localised PCA. Some followed patients prospectively and compared treatments, but did not include all the major treatments for PCA in their cohort, or did not collect the data in such a way that each aspect of treatment could be compared separately. ${ }^{13-15}$ For this reason, the adverse effects over time of HT may not have become apparent. A Dutch study compared the psychological impacts of RP and EBRT in patients with PCA. ${ }^{16}$ Participants were recruited before the commencement of treatment, when they were classified as having either high or low pretreatment anxiety. The authors observed that, within the group classified as having high pretreatment anxiety, levels of depression (but not anxiety) at 5-year follow-up were greatest in those who had received EBRT. ${ }^{16}$ A 2007 United States study followed a cohort of patients with localised PCA over 2 years and found no significant differences between groups treated with RP, brachytherapy or EBRT in terms of the mental and physical domains of the SF-36. ${ }^{17}$ Although a proportion of patients in the RP group were reported to have received adjuvant $\mathrm{HT}$, the authors did not report an analysis of HT outcomes. ${ }^{17}$

Only two studies to our knowledge have examined the specific effect of HT on HRQOL and psychological status in comparison to other treatments. One compared HRQOL between patients who received RP, brachytherapy or EBRT at nine US hospitals. ${ }^{18}$ The researchers documented the fact that some of the patients who received the radiotherapies also received adjuvant HT. Their measure of HRQOL included a category of "vitality or hormonal function" which incorporated aspects of depression, fatigue, weight change, gynaecomastia and hot flushes. They found that adjuvant HT was associated with lower vitality and worse hormonal function outcomes 24 months after commencement of PCA treatment. ${ }^{18}$ Another study also examined the contribution of HT, RP, EBRT and WW to patients' HRQOL several years after initial PCA treatment. ${ }^{19}$ This study employed a populationbased cross-sectional design using a cancer registry that identified all men in the southern part of the Netherlands who were diagnosed with PCA over a 4-year period, and that included information about the PCA treatment the men received. A comparison of patients who had received different PCA treatments more than 6 years earlier showed that HT was associated with the worst vitality outcome of all the treatment groups, with $\mathrm{RP}$ rating the best, followed by WW and then EBRT.

Although these two studies suggest poorer psychological outcomes with HT, it should be noted that neither used a specific measure of psychological status equivalent to the BSI-53 in our study, relying instead on the mental health components of general HRQOL measures. Nevertheless, these findings and ours may be pointing to an important role for physiological testosterone levels in maintaining optimal cognitive and emotional functioning of the male human brain. Alternatively, or in addition, the findings may point to mens' psychological response, over time, to the side effects of medical sex hormone manipulation — reduced libido, feminising physical changes (eg, reduced facial hair growth, loss of muscle bulk, gynaecomastia and skin changes), and unfamiliar and distressing physical symptoms (eg, hot flushes). ${ }^{4}$

We believe that the perception that men generally cope well with the diagnosis of PCA and its subsequent treatment is challenged by these emerging findings. A social expectation of outer masculine fortitude in the face of a cancer diagnosis, and a possible reluctance by clinicians to assess thoroughly their male patients' psychological status may be preventing cancer clinicians from gaining a fuller understanding of the factors that should be considered when recommending a particular treatment option to a patient newly diagnosed with PCA. Furthermore, such medical attitudes may be preventing patients from being offered necessary psychological and psychiatric support once they commence treatment for PCA.

There are several limitations of our study. First, recruitment of patients through cancer clinics introduces a possible selection bias, as not all men with PCA in the community had an equal chance of being recruited. Although the use of cancer registries reduces selection bias, inevitable delays between diagnosis and inclusion in the register preclude access during the critical period when patients are grappling with the implications of a cancer diagnosis. Accurate and reliable assessment of psychological adjustment to PCA clearly requires measuring psychosocial variables in real time. Recruitment through advertisements or from information sessions and self-help groups is prone to 


\section{DEPRESSION AND ANXIETY WITH PHYSICAL ILLNESS}

even greater bias because participants selfselect. For these reasons, using consecutive clinic attendees is common in psychooncology research.

A second limitation is attrition, but a substantial proportion of patients - nearly $90 \%$ - participated in the follow-up. Finally, we recruited English-speaking men only, so there are limits to how far we can generalise our results to non-English-speaking groups in the community.

We acknowledge that multiple factors (such as age, marital status, previous medical history) could influence the treatment the doctor recommends, whether the patient accepts the recommendation, and the ultimate psychological outcome for the patient. This is an important area for future research.

In summary, our findings suggest that psychological distress was greater with HT than with RP and OET, neither of which had distress levels significantly different from that with WW 12 months after PCA treatment commenced. Perhaps HT is less well tolerated by patients, but further research is needed to ascertain the reasons for our observation. Future studies should examine separately the psychosocial impact of HT, and explore whether there are mediating factors. The implications for counselling, psychological and psychiatric support are clear - clinicians should be particularly diligent in monitoring men receiving HT so that any adverse psychological effects may be promptly identified and treated.

\section{ACKNOWLEDGEMENTS}

Our study was supported by grants from the National Health and Medical Research Council (NHMRC) and the Bethlehem Griffiths Research Foundation. These funding bodies had no role in study design, data collection, analysis, interpretation or writing of this article. We would like to thank all of the patients who kindly participated in this study and the clinicians who recruited them.

\section{COMPETING INTERESTS}

None identified.

\section{AUTHOR DETAILS}

Jeremy W Couper, MB BS, MMed(Psych), MD, Senior Lecturer, Department of Psychiatry, ${ }^{1}$ and Head, Department of Psychiatry ${ }^{2}$

Anthony W Love, MA(Clin Psych), PhD, Professor of Psychology ${ }^{3}$

Judith V Dunai, MPsych(Clin), PhD, Clinical

Psycholgist and Research Assistant,

Department of Clinical Psychology ${ }^{2}$

Gillian M Duchesne, BSc(Hons), MB ChB, MD,

Professor, ${ }^{1}$ and Director of Radiation Oncology ${ }^{2}$

Sidney Bloch, MB ChB, PhD, Professor,

Department of Psychiatry and Centre for Health and Society ${ }^{1}$

Anthony J Costello, MB BS, MD, Director of Urology 4

David W Kissane, MB BS, MPM, MD, Alfred P.

Sloan Chair, Attending Psychiatrist and

Chairman, ${ }^{5}$ and Professor of Psychiatry ${ }^{6}$

1 University of Melbourne, Melbourne, VIC.

2 Peter MacCallum Cancer Centre, Melbourne,

VIC.

3 University of Ballarat, Mt Helen Campus, Ballarat, VIC

4 Royal Melbourne Hospital, Melbourne, VIC

5 Department of Psychiatry and Behavioral

Sciences, Memorial Sloan-Kettering Cancer

Center, New York, NY, USA.

6 Weill Cornell Medical College, New York, NY, USA.

Correspondence:

jeremy.couper@petermac.org

\section{REFERENCES}

1 Bloch S, Love A, Macvean M, et al. Psychological adjustment of men with prostate cancer: a review of the literature. Biopsychosoc Med 2007; $1: 2$.

2 Australian Institute of Health and Welfare and Australasian Association of Cancer Registries. Cancer in Australia 2001. Canberra: AlHW, 2004. (AlHW Catalogue No. CAN 23. Cancer Series No. 28.) http://www.aihw.gov.au/publications/can/ca01/ca01.pdf (accessed Feb 2009).

3 Barry MJ, Kaufman DS, Wu C-L. Case 15-2008: a 55-year-old man with an elevated prostatespecific antigen level and early-stage prostate cancer. N Engl J Med 2008; 358: 2161-2168.

4 Wilt TJ, Thompson IM. Clinically localised prostate cancer. BMJ 2006; 333: 1102-1106.

5 Couper JW. The effects of prostate cancer on intimate relationships. J Mens Health Gend 2007: 4: 226-232.
6 Love AW, Scealy M, Bloch S, et al. Psychosocial adjustment in newly diagnosed prostate cancer. Aust N Z J Psychiatry 2008; 42: 423-429.

7 Ware JE, Sherborne CD. The MOS 36-Item Short-Form Health Survey (SF-36). I. Conceptual framework and item selection. Med Care 1992; 30: 473-483.

8 McHorney CA, Ware JE, Raczek AE. The MOS 36-Item Short-Form Health Survey (SF-36). II. Psychometric and clinical tests of validity in measuring physical and mental health constructs. Med Care 1993; 31: 247-263.

9 Derogatis LR. BSI brief symptom inventory. Administration, scoring, and procedures manual. 4th ed. Minneapolis: National Computer Systems, 1993.

10 Derogatis LR, Melisaratos N. The brief symptom inventory: an introductory report. Psychol Med 1983; 13: 595-605.

11 Kissane DW, Bloch S. Family focused grief therapy. Buckingham: Open University Press, 2002.

12 Couper JW, Bloch S, Love A, et al. The psychosocial impact of prostate cancer on patients and their partners. Med J Aust 2006; 185: 428432.

13 Jayadevappa R, Chhatre S, Whittington R, et al. Health-related quality of life and satisfaction with care among older men treated for prostate cancer with either radical prostatectomy or external beam radiation therapy. BJU Int 2006; 97: 955-962.

14 Namiki S, Saito S, Tochigi T, et al. Impact of hormonal therapy before radical prostatectomy on the recovery of quality of life. Int J Urol 2005; 12: 173-181.

15 Yoshimura K, Arai Y, Ichioka K, et al. A 3-y prospective study of health-related and disease-specific quality of life in patients with nonmetastatic prostate cancer treated with radical prostatectomy or external beam radiotherapy. Prostate Cancer Prostatic Dis 2004; 7: 144-151.

16 Korfage IJ, Essink-Bot M-L, Janssens ACJW, et al. Anxiety and depression after prostate cancer diagnosis and treatment: 5-year follow-up. Br J Cancer 2006; 94: 1093-1098.

17 Litwin MS, Gore JL, Kwan L, et al. Quality of life after surgery, external beam irradiation, or brachytherapy for early-stage prostate cancer. Cancer 2007; 109: 2239-2247.

18 Sanda MG, Dunn RL, Michalski J, et al. Quality of life and satisfaction with outcome among prostate-cancer survivors. N Engl J Med 2008; 358: 1250-1261.

19 Mols F, van de Poll-Franse LV, Vingerhoets AJJM, et al. Long-term quality of life among Dutch prostate cancer survivors. Cancer 2006; 107: 2186-2196.

(Received 31 Jul 2008, accepted 29 Oct 2008) 